\title{
INERTIAL SUBALGEBRAS OF CENTRAL SEPARABLE ALGEBRAS
}

\author{
NICHOLAS S. FORD ${ }^{1}$
}

\begin{abstract}
Let $R$ be a commutative ring with 1 . An $R$-separable subalgebra $B$ of an $R$-algebra $A$ is said to be an $R$-inertial subalgebra provided $B+N=A$, where $N$ is the Jacobson radical of $A$. Suppose $A$ is a finitely generated $R$-algebra which is separable over its center $Z(A)$. We show that if $A$ possesses an $R$-inertial subalgebra $B$, then $Z(A)$ possesses a unique $R$ inertial subalgebra $S$. Moreover, $A$ can be decomposed as $A \simeq B \otimes_{S} Z(A)$. Suppose $C$ is a finitely generated, commutative, semilocal $R$-algebra with $R$ inertial subalgebra $S$. We show that the $R$-inertial subalgebras of each central separable $C$-algebra are unique up to an inner automorphism generated by an element in the radical of the algebra if and only if the natural mapping of the Brauer groups $\beta(S) \rightarrow \beta(C)$ is a monomorphism. We conclude by presenting a method which enables one to construct algebras which possess nonisomorphic inertial subalgebras.
\end{abstract}

0 . Introduction. All rings are assumed to be associative and to possess an identity element 1 . By an algebra $A$ over a commutative ring $R$ (an $R$-algebra $A$ ) we mean a ring $A$ together with a ring homomorphism from $R$ into $Z(A)$, the center of $A$. An $R$-algebra $A$ will be called central separable if it is separable over $R$ and $Z(A)=R$. When we say an $R$-algebra is finitely generated or projective, we mean it is finitely generated or projective as an $R$ module. The Jacobson radical of an $R$-algebra $A$ will be denoted by $\operatorname{rad} A$. Finally, by an $R$-inertial subalgebra $B$ of an $R$-algebra $A$ we mean an $R$ separable subalgebra $B$ of $A$ having the property that $B+\operatorname{rad} A=A$.

I. Structure. Let $A$ be a finitely generated $R$-algebra which is separable over its center. This section investigates the relationship between the $R$-inertial subalgebras of $A$ and the $R$-inertial subalgebras of its center.

LEMMA 1.1. Let $A$ be a finitely generated $R$-algebra which is separable over its center $C$. If $B$ is an R-inertial subalgebra of $A$, then $B \cdot C=A$.

Proof. The assertion follows at once by an application of the original Nakayama Lemma.

Received by the editors September 18, 1974 and, in revised form, January 26, 1976.

AMS (MOS) subject classifications (1970). Primary 13A20, 16A16, 16A48; Secondary 13H99, $16 \mathrm{~A} 32$.

Key words and phrases. Inertial subalgebra, separable algebra, Jacobson radical, Brauer group.

1 The author wishes to express his gratitude to Professor Edward C. Ingraham of Michigan State University for his guidance in the preparation of this paper. Thanks are also due to the referee whose suggestions considerably strengthened some of the results. 
THEOREM 1.2. Suppose $A$ is a finitely generated $R$-algebra which is separable over its center $C$. Then an $R$-subalgebra $B$ of $A$ is an $R$-inertial subalgebra of $A$ if and only if:

(1) $B$ is central separable over its center $S$;

(2) $S$ is the unique $R$-inertial subalgebra of $C$; and

(3) $A \simeq B \otimes_{S} C$ as $C$-algebras.

Proof. Only if. First, since $B$ is an $R$-inertial subalgebra of $A$, and therefore $R$-separable, it follows that $B$ is necessarily separable over its center $S$. Next, it is immediate from Lemma 1.1 that $S$ is included in $C$. To show $S$ to be an $R$-inertial subalgebra of $C$ it suffices to show that $C=S+\mathfrak{n}$, where $\mathfrak{n}$ $=\operatorname{rad} C$. Let $N=\operatorname{rad} A$. We have by Azumaya [2, Corollary to Theorem 9], that $C \cap N \subseteq$ n. Furthermore, a straightforward application of Nakayama's Lemma yields the opposite inclusion, so that $C \cap N=\mathrm{n}$. In addition, since $A$ is separable over $C, Z(A / N)=[C+N] / N$. Similarly, $Z(B / B \cap N)$ $=[S+B \cap N] / B \cap N$. The following chain of isomorphisms is then clear:

$$
[S+\mathfrak{n}] / \mathfrak{n} \simeq Z(B / B \cap N) \simeq Z(A / N) \simeq C / \mathfrak{n}
$$

Since the composite of these mappings is the identity, we conclude that $S+\mathrm{n}=C$. Being a direct summand of $A$, the algebra $C$ is finitely generated over $R$. Separable (hence inertial) subalgebras of a finitely generated algebra are themselves finitely generated (Sanders [10]). The uniqueness of $S$ then follows from Ingraham [7, Proposition 2.6], which states that any two finitely generated inertial subalgebras of a finitely generated, commutative algebra must coincide. Finally, we assert that the natural $C$-algebra mapping $\mu$ : $B \otimes_{S} C \rightarrow A$ induced by $\mu(b \otimes c)=b \cdot c$ is an isomorphism. Since $\mu$ is onto by Lemma 1.1, it suffices to show that $\mu$ is one-to-one. Let $\mathfrak{A}=C \cap \operatorname{Ker} \mu$. Since $B \otimes_{S} C$ is central separable over $C$, it follows that

$$
\operatorname{Ker} \mu=\mathfrak{U} \cdot\left(B \otimes_{S} C\right) .
$$

Now if $\alpha \in \mathfrak{A}$, then $0=\mu(\alpha)=\mu(\alpha \cdot 1)=\alpha \cdot \mu(1)=\alpha \cdot 1=\alpha$. Thus $\mathfrak{A}$ $=(0)$, and therefore $\mu$ is one-to-one.

If. We first show that $B$ is an $R$-inertial subalgebra of $B \otimes_{S} C$. Being a central separable $S$-algebra, $B$ is $S$-projective and so can be identified as the subalgebra $B \otimes_{S} S$ of $B \otimes_{S} C$. Inasmuch as $B \otimes_{S} C$ is central separable over $C$, we have that $\operatorname{rad}\left(B \otimes_{S} C\right)=\mathfrak{n} \cdot\left(B \otimes_{S} C\right)=B \otimes_{S} n$. Therefore

$$
B \underset{S}{\otimes} S+\operatorname{rad}(B \underset{S}{\otimes} C)=B \underset{S}{\otimes} S+B \underset{S}{\otimes} \mathrm{n}=B \underset{S}{\otimes} C .
$$

Clearly $B$ is separable over $R$ since, by assumption, $S$ is separable over $R$. Thus $B \otimes_{S} S$ is an $R$-inertial subalgebra of $B \otimes_{S} C$. It is well known that every endomorphism of a central separable algebra is an automorphism. Thus the existence of an isomorphism from $B \otimes_{S} C$ to $A$ implies that the homomorphism $\mu$, previously defined, is an isomorphism. Therefore $B=\mu\left(B \otimes_{S} S\right)$ is an $R$-inertial subalgebra of $A$. 
II. Uniqueness and the Brauer group. We say that the uniqueness statement holds for the $R$-inertial subalgebras of an $R$-algebra $A$ if corresponding to each pair $B$ and $B^{\prime}$ of $R$-inertial subalgebras of $A$ there is an element $\eta \in \operatorname{rad} A$ such that $B^{\prime}=(1-\eta) B(1-\eta)^{-1}$. Suppose $C$ is a finitely generated, commutative $R$-algebra with $R$-inertial subalgebra $R$. We will show that under certain conditions, saying that the uniqueness statement holds for the $R$-inertial subalgebras of every central separable $C$-algebra is equivalent to saying that the natural mapping of the Brauer groups $\beta(R) \rightarrow \beta(C)$ is a monomorphism.

Proposition 2.1. If $A$ is a finitely generated R-algebra with $R$-inertial subalgebra $B$, then every central idempotent of $A$ is contained in $B$.

Proof. Let $e \in A$ be a central idempotent. It is easy to see that the subalgebra $B^{\prime}=B e \oplus B(1-e)$ is $R$-separable. Clearly $B \subseteq B^{\prime}$. Thus $B^{\prime}$ $+\operatorname{rad} A=A$, which shows that $B^{\prime}$ is an $R$-inertial subalgebra of $A$. Since $A$ is finitely generated over $R$, so are $B$ and $B^{\prime}$ (Sanders [10]). Moreover, finitely generated and nested inertial subalgebras necessarily coincide (Ingraham [7, Lemma 2.5]). Thus $B=B^{\prime}$, and $e \in B$.

A commutative ring is said to be semilocal if it contains only a finite number of maximal ideals. If a commutative ring has no idempotents but 0 and 1 , it is said to be connected. We will denote the Brauer group of a commutative ring $R$ by $\beta(R)$ (Auslander and Goldman [1]). If $C$ is a commutative $R$-algebra, there is a natural homomorphism: $\beta(R) \rightarrow \beta(C)$ defined by $[A] \rightarrow\left[A \otimes_{R} C\right]$. We will denote the kernel of this mapping by $\beta(C / R)$.

THEOREM 2.2. Suppose $R$ is a semilocal ring and $C$ is a finitely generated, faithful, commutative $R$-algebra with $R$ an $R$-inertial subalgebra of $C$. Then the uniqueness statement holds for the $R$-inertial subalgebras of every central separable C-algebra if and only if $\beta(R) \rightarrow \beta(C)$ is a monomorphism.

Proof. It is well known that finitely generated, commutative, semilocal rings may be decomposed as a finite direct sum of connected semilocal rings. Thus we may write $C=\oplus \sum_{i=1}^{n} C_{i}$, where each $C_{i}$ is connected. This in turn induces a decomposition on any $C$-algebra $A$ as $\oplus \sum_{i=1}^{n} A_{i}$, where each $A_{i}$ is (central) separable over $C_{i}$ if and only if $A$ is (central) separable over $C$. Moreover, in view of Proposition 2.1, $R$ may be decomposed as $\oplus \sum_{i=1}^{n} R_{i}$, where each $R_{i}$ is connected, semilocal, and an $R_{i}$-inertial subalgebra of $C_{i}$. In this context it is straightforward to verify the following two assertions.

(1) The uniqueness statement holds for the $R$-inertial subalgebras of every central separable $C$-algebra if and only if it holds for the $R_{i}$-inertial subalgebras of every central separable $C_{i}$-algebra, for every $i \leqslant n$.

(2) The natural mapping of the Brauer groups $\beta(R) \rightarrow \beta(C)$ is a monomorphism if and only if the natural mapping of the Brauer groups $\beta\left(R_{i}\right) \rightarrow \beta\left(C_{i}\right)$ is a monomorphism, for every $i \leqslant n$.

In light of this we may assume, without loss of generality, that $C$ is a connected $R$-algebra. Let us first suppose that the uniqueness statement holds 
for all central separable $C$-algebras. It is well known that finitely generated and projective modules over a connected and semilocal ring are free of finite rank. Hence if $[B] \in \beta(C / R)$ then $B \otimes_{S} C \simeq M_{n}(C)$, where $M_{n}(C)$ denotes the $n \times n$ matrix algebra over $C$. Since the subalgebras $B$ and $M_{n}(R)$ of $M_{n}(C)$ are central separable over $R$, it follows from Theorem 1.2 that each is an $R$ inertial subalgebra of $M_{n}(C)$. Thus $B \simeq M_{n}(R)$ by assumption, so that $\beta(R) \rightarrow \beta(C)$ is a monomorphism.

Conversely, suppose $\beta(R) \rightarrow \beta(C)$ is a monomorphism. Let $B$ and $B^{\prime}$ be $R$ inertial subalgebras of a central separable $C$-algebra $A$. Then

$$
B \otimes_{R} C \simeq B^{\prime} \otimes_{R} C
$$

by Theorem 1.2 , so that $\left[B \otimes_{R} C\right]=\left[B^{\prime} \otimes_{R} C\right]$ in $\beta(C)$. By hypothesis, this implies that $[B]=\left[B^{\prime}\right]$ in $\beta(R)$. Since $R$ is semilocal and connected it follows by DeMeyer [4, Corollary 1$]$, that there exists a representative $D \in[B]$ unique up to isomorphism, possessing no idempotents except 0 and 1 , such that $B \simeq M_{u}(D)$ and $B^{\prime} \simeq M_{t}(D)$ as $R$-algebras for uniquely determined integers $u$ and $t$. Thus $M_{u}(D) \otimes_{R} C \simeq M_{t}(D) \otimes_{R} C$ and, since $D \otimes_{R} C$ is a central separable $C$-algebra, dimensionality arguments allow us to conclude that $u=t$. Hence there is an isomorphism from $B$ to $B^{\prime}$ which we denote by $f$. We note that the mapping $f \otimes 1: B \otimes_{R} C \rightarrow B^{\prime} \otimes_{R} C$ induced by

$$
f \otimes 1(b \otimes c)=f(b) \otimes c
$$

is a $C$-algebra isomorphism. In view of Theorem 1.2, the multiplication maps $\mu: B \otimes_{R} C \rightarrow A$ and $\mu^{\prime}: B^{\prime} \otimes_{R} C \rightarrow A$ are also $C$-algebra isomorphisms. Therefore by [9], Roy and Sridharan's generalization ${ }^{2}$ of the Skolem-Noether Theorem, there exists an inner automorphism $\theta$ of $A$ such that $\mu=\theta$ $\circ\left(\mu^{\prime} \circ f \otimes 1\right)$. It follows that

$$
B=\mu\left(B \otimes_{R} R\right)=\theta\left(\mu^{\prime}\left(f \otimes 1\left(B \otimes_{R} R\right)\right)\right)=\theta\left(\mu^{\prime}\left(B^{\prime} \otimes_{R} R\right)\right)=\theta\left(B^{\prime}\right) .
$$

Suppose $\theta(x)=w x w^{-1}$. Since $A=B+N$, where $N=\operatorname{rad} A$, we can represent $w$ as $w=v+\eta$ where $v \in B$ and $\eta \in N$. Now $w v^{-1}=1+\eta v^{-1}$, and so is of the form $1-r$ where $r \in N$. Then

$$
\left(w v^{-1}\right) B\left(w v^{-1}\right)^{-1}=w\left(v^{-1} B v\right) w^{-1}=w B w^{-1}=B^{\prime} .
$$

Azumaya has shown [2] that the uniqueness statement holds for the inertial subalgebras of any finitely generated algebra over a Hensel ring. It is by no means true that the uniqueness statement holds in general. In fact, as we are about to show, the inertial subalgebras of an algebra need not even be isomorphic.

Let $R$ be a local (noetherian) domain with maximal ideal $m$. Let $S$ be an

2 The hypothesis in [9] that the ground ring is noetherian may be deleted without altering the conclusion. 
extension of $R$ which is finitely generated as an $R$-module. Define the (finitely generated) $R$-subalgebra $C$ of $S$ by $C=R+I$, where $I=\mathfrak{m} \cdot S$. We see that $I^{2} \subseteq \mathrm{m} \cdot C \subseteq \operatorname{rad} C$. Moreover, $C / I \simeq R / \mathrm{m}$. Hence $I=\operatorname{rad} C$, showing $C$ to be a local ring with maximal ideal $I$.

Theorem 2.3. Let $R$ be an integrally closed, local, noetherian domain with maximal ideal $\mathfrak{m} \neq 0$. Suppose $\beta(R) \neq 0$, and that the quotient field of $R$ is a finite algebraic number field. Then for each nontrivial representative $A \in \beta(R)$ there exists a finitely generated, commutative $R$-algebra $C$ such that $A \otimes_{R} C$ has nonisomorphic $R$-inertial subalgebras.

Proof. For each (nontrivial) representative $A \in \beta(R)$ there exists a finitely generated, free, commutative, and separable splitting algebra $S$ (Auslander and Goldman [1, Theorem 6.3]). Since $S$ may be assumed to be connected without loss of generality, it follows from Janusz [8, Corollary 4.2], that $S$ is, moreover, an integral domain. Define $C=R+\mathfrak{m} \cdot S$. Since $m \neq 0$, the quotient field of both $S$ and of $C$ is $L=Q(S)$, where $Q$ is the rational number field. One then obtains the following commutative diagram:

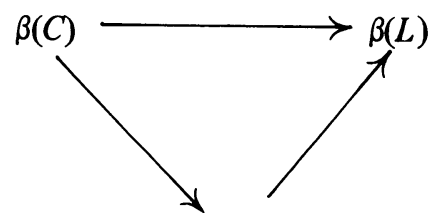

$\beta(S)$

Since $L$ is a finite algebraic number field, it follows from Childs [3, Corollary 4.3], that the mappings $\beta(C) \rightarrow \beta(L)$ and $\beta(S) \rightarrow \beta(L)$ are one-to-one. Thus $\beta(C) \rightarrow \beta(S)$ is one-to-one, so that $\beta(S / R)=\beta(C / R)$. Therefore $A$ $\in \beta(C / R)$ and, inasmuch as $C$ is local, $A \otimes_{R} C \simeq M_{n}(C)$ for some integer $n$. We see, by Theorem 1.2, that both $A$ and $M_{n}(R)$ are $R$-inertial subalgebras of $A \otimes_{R} C$. However, by its definition, $A$ cannot be isomorphic to $M_{n}(R)$.

COROllaRy 2.4. There exist local extensions $C$ of $R$ which contain $R$ as an $R$ inertial subalgebra, and which afford matrix algebras over $C$ possessing nonisomorphic $R$-inertial subalgebras.

Corollary 2.5. There exist local extensions $C$ of $R$ which have the same residue class field as $R$, but such that $\beta(R) \rightarrow \beta(C)$ is not a monomorphism.

A specific example where this phenomenon occurs is: $R=Z_{(5)}, C=Z_{(5)}$ $\oplus i 5 Z_{(5)}, S=Z_{(5)} \oplus i Z_{(5)}$, and $A=$ the quaternion algebra over $Z_{(5)}$; where $i^{2}=-1$. A discussion of generalized quaternion algebras may be found in Dickson [5]. 


\section{REFERENCES}

1. M. Auslander and $\mathrm{O}$. Goldman, The Brauer group of a commutative ring, Trans. Amer. Math. Soc. 97 (1960), 367-409. MR 22 \# 12130.

2. G. Azumaya, On maximally central algebras, Nagoya Math. J. 2 (1951), 119-150. MR 12, 669.

3. L. N. Childs, Mayer-Vietoris sequences and Brauer groups of nonnormal domains, Trans. Amer. Math. Soc. 196 (1974), 51-67. MR 49 \#8979.

4. F. R. DeMeyer, Projective modules over central separable algebras, Canad. J. Math. 21 (1969) 39-43. MR 38 \#3299.

5. L. E. Dickson, Algebras and their arithmetic, Dover, New York, 1960. MR 22 \#2625.

6. S. Endo and Y. Watanabe, On separable algebras over a commutative ring, Osaka J. Math. 4 (1967), 233-242. MR 37 \#2796.

7. E. C. Ingraham, Inertial subalgebras of algebras over commutative rings, Trans. Amer. Math. Soc. 124 (1966), 77-93. MR 34 \#209.

8. G. J. Janusz, Separable algebras over commutative rings, Trans. Amer. Math. Soc. 122 (1966), 461-479. MR 35 \# 1585.

9. A. Roy and R. Sridharan, Derivations in Azumaya algebras, J. Math. Kyoto Univ. 7 (1967), 161-167.

10. D. Sanders, The dominion and separable subalgebras of finitely generated algebras, Proc. Amer. Math. Soc. 48 (1975), 1-7.

Department of Mathematics, Pennsylvania State University, Fayette Campus, Uniontown, Pennsylvania 15401 